\title{
Value of information: facilitating targeted information acquisition in decision processes
}

\author{
Jeffrey M. Keisler
}

Published online: 19 February 2014

(c) Springer Science+Business Media New York 2014

ESD's special issue on the value of information (VoI) is the first one in the history of this relatively old concept that is now emerging as a focus of analysis in its own right. Information is amorphous, and valuing it seems a challenge akin to weighing the water vapor in a cloud. When the cloud meets colder air and rain falls, then we can measure the depth and area of the rainfall. Analogously, when information meets decisions, we can measure how the information changes the value resulting from the better outcomes arising from more appropriate choices, and that change in value is the value due to the information.

Over 50 years ago, solid decision theoretical results on VoI were already known (e.g., Raiffa and Schlaifer 1961). That such a simple formulation works on such a vexing problem is elegant and almost amazing. When information is scarce, knowing its value allows decision makers to determine when to expend resources to obtain it.

Due to all sorts of technological advances, options for acquiring information are relatively abundant today compared with what they were 50 years ago. Furthermore, improved computational power means that information once acquired can be incorporated into ever more complex decisions. But acquiring information is still not cheap, and the multiplicity of options only means that there are more decisions about what information to acquire. Thus, VoI methods belong in the toolkit of modelers, analysts and policy makers.

This special issue highlights the importance of these methods and the range of ways they are advancing in

J. M. Keisler ( $₫)$

College of Management, University of Massachusetts Boston,

Boston, MA 02125, USA

e-mail: jeff.keisler@umb.edu sophistication and function. First, Keisler et al. (2014) survey the last decade of practice and find substantial growth, with areas and forms of application evolving in line with the change in types of problems decision makers face and the analytical tools and information sources to be utilized.

Because information can take so many forms, highly general mathematical results about the nature of its value are notoriously difficult to obtain. However, it is possible to gain insights and create a solid base for improved analytical tools for families of applications by starting with small sets of basic assumptions and deriving results that hold whenever those assumptions hold. Sun and Abbas (2014) develop fundamental results relating problem characteristics to decision maker characteristics. Specifically, they obtain powerful results about the relationship between VoI and decision makers' risk attitudes. Following this, Bakir and Klutke (2014) consider a class of problems in which information acquisition can focus on separate events. Their results provide a way to determine where to obtain the comparative value of different information acquisition strategies focused around the drivers of different events.

Masinter et al. (2014) consider research as an information source which can fill gaps in knowledge. Their framework represents the state of knowledge about an issue as a network of hypotheses, where research can confirm or reject the hypotheses. Such decision problems are common in developing scientific areas, such as the area of nanotechnology analyzed in this paper's example. Rehr et al. (2014) likewise consider VoI as a way to structure and improve scientific debate. They develop a framework that takes account of how scientific studies would affect stakeholder views in concrete decision contexts. They illustrate with a case involving environmental decisions affecting a marine ecosystem. 
In other situations, the challenging part of the decision problem is the technical nature of the information itself, and how that information functions within the analysis of a technically advanced model. Solving such problems also expands the theoretical toolkit. Gradowska and Cooke (2014) develop a model where information feeds into decisions that may reduce health risks resulting from fish consumption. The risk model here has a complex and welldocumented scientific structure, but with uncertainty about the effects of particular mechanisms. By combining VoI with Bayes' net modeling, they develop a way to synthesize existing judgment along with acquisition of new information about many interacting issues in a single problem. Cooke et al. (2014) consider space-borne climate observing systems, where, again, information feeds a rich scientific model and subsequently rich economic model that can generate predictions and ultimately guide highly important policy decisions. Information here is not acquired in easily separable units. Rather, it is necessary to invest in a whole system that can generate a large amount of data, to sense conditions of and changes in conditions of numerous components of the planet's climate. In addressing this problem, the authors provide an approach to relate the rich body of information that a system can generate to the rich model it will inform, so as to understand the system's potential benefit. In part, this involves identifying uncertainties about specific physical parameters within scientific models and how powerful the system will be in reducing those uncertainties.

Of interest in connecting VoI models to practical decision making, Le et al. (2014) start with a parametric flow model for managing water pollution. This application, for a particular important water system, demonstrates the type of choices modelers must make to adapt even parametric models to the variations presented by real problems and real constraints that decision makers often face. Working out these details allows the potential of a theoretical model to be realized in multiple instances and locations.

Finally, Trainor-Guitton (2014) incorporates VoI into geophysically driven problems. The methods developed here utilize remote sensing in conjunction with experiments and other data sources and feed all these data into sophisticated geologic models. In this situation, some data are easily acquired in large amounts, but there are significant constraints to acquiring useful information about many of the parameters that are key to decision models. The examples show how VoI produces insights into the improvement of aquifer management-an area with economic, societal and environmental importance. These methods extend naturally to other geological applications, such as energy production. Like the other applications in this issue, the multi-level modeling approach can guide credible decision making that manages large impacts in the face of knowledge gaps, technical complexity.
This spectrum of activity, from basic theory to tools to specific applications, gives a feel for how VoI as a concept is developing as both art and science. As we face decisions with ever greater impact, methods like the ones described in this issue can help realize the potential for improving those decisions by making use of the myriad ways we might invest in obtaining information. This work shows a path for constructing sophisticated decision models that connect advanced knowledge from the scientific disciplines with understanding of the real-world impact of human actions.

In spite of this potential and the promising applications we see, it is still uncommon for VoI to be explicitly considered in important decisions. As organizations and even humanity itself act on ever larger scales, high-quality decisions are critical and high-quality information is not always available. This is the time for VoI to help bridge this gap. Researchers and practitioners working with value of information are finding it an area ripe for rapid progress, one where they can produce developments and discoveries that make the world's decisions better and can thereby make the world better.

\section{References}

Bakır NO, Klutke G-A (2014) Buying price of event information in two-action decision problems. Environ Syst Decis 34(1). doi:10. 1007/s10669-013-9478-x

Cooke R, Wielicki B, Young D, Mlynczak M (2014) Value of information for climate observing systems. Environ Syst Decis 34(1). doi:10.1007/s10669-013-9451-8

Gradowska P, Cooke R (2014) Estimating expected value of information using Bayesian belief networks: a case study in fish consumption advisory. Environ Syst Decis 34(1). doi:10.1007/ s10669-013-9471-4

Keisler JM, Collier ZA, Chu E, Sinatra N, Linkov I (2014) Value of information analysis: the state of application. Environ Syst Decis 34(1). doi:10.1007/s10669-013-9439-4

Le AH, Tokai A, Nakakubo T (2014) Applying value of information methods to prioritize elements for water quality management with an example of linear alkylbenzene sulfonate in the Yodo River, Japan. Environ Syst Decis 34(1). doi:10.1007/s10669014-9490-9

Masinter A, Small M, Casman E (2014) Research prioritization using hypothesis maps. Environ Syst Decis 34(1). doi:10.1007/s10669014-9489-2

Raiffa H, Schlaifer R (1961) Applied statistical decision theory. Division of Research, Harvard Business School, Boston

Rehr A, Small MJ, Fischbeck PS, Bradley P, Fisher WS (2014) The role of scientific studies in building consensus in environmental decision making: a coral reef example. Environ Syst Decis 34(1). doi:10.1007/s10669-014-9491-8

Sun Z, Abbas AE (2014) On the sensitivity of the value of information to risk aversion in two-action decision problems. Environ Syst Decis 34(1). doi:10.1007/s10669-013-9477-y

Trainor-Guitton WJ (2014) A geophysical perspective of value of information: examples of spatial decisions for groundwater sustainability. Environ Syst Decis 34(1). doi:10.1007/s10669013-9487-9 